\title{
04.1
}

\section{Два вида структуры плазменного канала в импульсном разряде высокого давления в цезии}

\author{
(C) Ф.Г. Бакшт ${ }^{1}$, В.Ф. Лапшин ${ }^{1,2, \text { Ф }}$ \\ ${ }^{1}$ Физико-технический институт им. А.Ф. Иофффе РАН, Санкт-Петербург, Россия \\ 2 Петербургский государственный университет путей сообщения Императора Александра І, Санкт-Петербург, Россия \\ ฯ E-mail: lapshinvf@mail.ru
}

Поступило в Редакцию 23 июля 2021 г.

В окончательной редакции 23 июля 2021 г.

Принято к публикации 14 августа 2021 г.

\begin{abstract}
На основе уравнений радиационной газодинамики выполнено моделирование импульсно-периодического разряда высокого давления в парах цезия. Показано, что в разряде возможна реализация двух различных видов структуры плазменного канала. В начале импульса тока плазменный канал разряда имеет центрированную структуру. При этом бо́льшая часть плазмы сосредоточена вблизи оси разряда. Концентрация заряженных частиц убывает вдоль радиуса. Затем, если амплитуда тока достаточно велика, в процессе нагрева плазмы происходит трансформация от центрированной к оболочечной структуре канала. При этом бо́льшая часть плазмы оказывается сосредоточенной на периферии разряда и ее концентрация возрастает вдоль радиуса от оси к стенкам трубки. Показано, что переход от одной структуры канала к другой происходит в момент времени, когда удельная теплоемкость плазмы вблизи оси достигает глубокого минимума, соответствующего полностью однократно ионизованной $e-i$-плазме.
\end{abstract}

Ключевые слова: низкотемпературная плазма, импульсный разряд, плазменный канал.

DOI: 10.21883/PJTF.2021.22.51727.18972

Импульсный газовый разряд высокого давления, ограниченный твердыми стенками, широко используется для создания эффективных интенсивных источников ультрафиолетового, видимого и инфракрасного излучения. При описании таких разрядов ранее в литературе рассматривались режимы горения, в которых плазменный канал имеет простейшую центрированную структуру, когда бо́льшая часть плазмы сосредоточена в приосевой области [1-5]. На различные формы плазменного канала в импульсно-периодическом разряде (ИПР) высокого давления в цезии указывалось в последних работах [6-9]. В настоящей работе на примере ИПР в цезии рассматривается трансформация структуры плазменного канала разряда от центрированной к оболочечной форме в процессе нагрева плазмы импульсом тока.

В работе исследуется разряд в длинной керамической трубке из $\mathrm{Al}_{2} \mathrm{O}_{3}$ с внутренним радиусом $R=2 \mathrm{~mm}$. Рассматривается установившийся режим горения, когда через плазму „дежурного“ разряда, поддерживаемого постоянным током $I_{0}=1.0 \mathrm{~A}$, периодически (с частотой $v=1400 \mathrm{~Hz}$ ) пропускается импульс тока заданной амплитуды $I_{\max }=100$ А и длительности $t_{p}=50 \mu \mathrm{s}$. Количество цезия в трубке определяется давлением насыщающих паров $p_{s a t}$ у наиболее холодного конца трубки. При значениях $p_{\text {sat }}=75-500$ Torr давление плазмы в импульсе достигает значений $p=400-1200$ Torr, температура стенок газоразрядной трубки имеет значения $T_{w}=1300-1500 \mathrm{~K}$, а температура плазмы на оси достигает $17000 \mathrm{~K}$. Плазма с такими параметрами находится в состоянии локального термодинамического равновесия, и для ее описания применимы уравнения
Саха-Больцмана. Для моделирования ИПР в цезии используются уравнения радиационной газодинамики, записанные с учетом существенно дозвуковых скоростей движения плазмы и аксиальной симметрии разряда:

$$
\begin{gathered}
\frac{\partial}{\partial t}\left(n_{a}+n_{i}\right)+\frac{1}{r} \frac{\partial}{\partial r}\left[r\left(n_{a} V_{a}+n_{i} V_{i}\right)\right]=0, \\
n_{e} n_{1}=K_{1}(T) n_{a}, n_{e} n_{2}=K_{2}(T) n_{1}, n_{e} n_{3}=K_{3}(T) n_{2}, \\
\frac{\partial}{\partial r} p_{a}=n_{a} n_{1}\left(V_{i}-V_{a}\right) r_{a i}, \\
\frac{\partial}{\partial r}\left(p_{e}+p_{i}\right)=n_{1} n_{a}\left(V_{a}-V_{i}\right) r_{a i}, \\
\frac{\partial}{\partial t}\left[\frac{3}{2}\left(n_{a}+n_{i}+n_{e}\right) k_{\mathrm{B}} T+n_{a} E_{a}+n_{i} E_{i}\right] \\
+\frac{1}{r} \frac{\partial}{\partial r}\left\{r \left[\frac{5}{2} k_{\mathrm{B}} T\left(n_{e} V_{e}+n_{a} V_{a}+n_{i} V_{i}\right)\right.\right. \\
\left.\left.+E_{a} n_{a} V_{a}+E_{i} n_{i} V_{i}\right]\right\}=\sigma E^{2}+\frac{1}{r} \frac{\partial}{\partial r} r \lambda \frac{\partial T}{\partial r}-W_{r a d}, \\
I(t)=2 \pi E_{z}(t) \int_{0}^{R} r \sigma_{e}(r, t) d r .
\end{gathered}
$$

Здесь $r$ - радиальная координата, $k_{\mathrm{B}}-$ постоянная Больцмана, $T$ - температура, $n_{\alpha}, V_{\alpha}, p_{\alpha}=n_{\alpha} k_{\mathrm{B}} T-$ соответственно концентрация, радиальная скорость и парциальное давление компоненты плазмы сорта $\alpha$ $(\alpha=a, i, e), \quad n_{1}, \quad n_{2}$ и $n_{3}-$ концентрации одно-, двух- и трехкратно ионизованных атомов цезия, 
$n_{e}=n_{1}+2 n_{2}+3 n_{3}, \quad n_{i}=n_{1}+n_{2}+n_{3}, \quad E_{a}-$ энергия возбуждения атомов, усредненная по распределению Больцмана, $n_{i} E_{i}=n_{1} E_{1}+n_{2}\left(E_{1}+E_{2}\right)+n_{3}\left(E_{1}+E_{2}+E_{3}\right)$, $E_{1}=3.89 \mathrm{eV}, E_{2}=23.15 \mathrm{eV}, E_{3}=33.4 \mathrm{eV}$ - энергии первой, второй и третьей ионизации атомов цезия [10], $E_{z}-$ напряженность продольного электрического поля в плазме, $K_{1}(T), K_{2}(T)$ и $K_{3}(T)$ - константы равновесия из соотношения Саха $[11,12]$. В случае частично ионизованной плазмы для расчета коэффициентов электропроводности $\sigma_{e}$, теплопроводности $\lambda$ и трения $r_{a i}$ использовалась методика из работы [3]. В полностью ионизованной плазме, содержащей ионы различной кратности, для $\lambda$ и $\sigma_{e}$ использовались выражения из работы [13].

Предполагается, что радиальный ток на стенки газоразрядной трубки отсутствует и радиальные скорости электронов и всех видов ионов одинаковы: $V_{i}=V_{e}$. В работе рассматривается слаботочный разряд, в котором эффектом самосжатия разряда собственным магнитным полем можно пренебречь.

Значения радиационных потерь энергии из плазмы $W_{\text {rad }}$ рассчитывались методом прямого интегрирования уравнения переноса излучения [14]. При этом коэффициент поглощения $k_{\lambda}$, соответствующий процессам фотоионизации возбужденных состояний ионов цезия, рассчитывался в водородоподобном приближении [11]. Во всех остальных случаях при вычислении $k_{\lambda}$ использовалась методика из работы [3].

Граничные условия к уравнениям (1)-(6) при $r=0$ соответствуют условию аксиальной симметрии плазмы. При записи граничных условий на стенке трубки учтено, что в установившемся режиме горения ИПР масса цезия $M$, приходящаяся на единицу длины трубки, остается постоянной в течение всего периода импульса тока

$$
M=2 \pi m_{a} \int_{0}^{R} r\left(n_{a}+n_{i}\right) d r=\text { const. }
$$

Здесь $m_{a}$ - масса атома цезия. Температура плазмы $T(R)$ предполагается равной температуре стенки трубки $T_{w}$ и определяется соотношением [6]:

$$
\begin{aligned}
T(R) & =T_{w}=\left(\frac{q_{p l}+q_{r a d}}{\varepsilon_{w} \sigma_{\mathrm{SB}}(1+\Delta R / R)}\right)^{1 / 4} \\
& +\frac{R}{\lambda_{w}} \ln \left(1+\frac{\Delta R}{R}\right)\left(q_{p l}+a q_{r a d}\right) .
\end{aligned}
$$

Здесь $\Delta R$ - толщина стенки (в расчетах использовалось $\Delta R=1.5 \mathrm{~mm}), \quad \lambda_{w}=13 \mathrm{~W} \cdot \mathrm{m}^{-1} \cdot \mathrm{K}^{-1}$ и $\varepsilon_{w}=0.3-$ теплопроводность и интегральная степень черноты сапфира [15], $\sigma_{\mathrm{SB}}$ - постоянная Стефана-Больцмана, $a=0.5\left[1 / \ln (1+\delta)-1 /\left(\delta+0.5 \delta^{2}\right)\right]$, $\delta=\Delta R / R$ $q_{p l}=-\left.v \int_{0}^{t_{p e r}}\left(\lambda \frac{\partial T}{\partial r}\right)\right|_{r=R} d t-$ средний за период поток тепла из плазмы на внутреннюю поверхность стенки газоразрядной трубки вследствие теплопроводности, $t_{p e r}=1 / v, q_{r a d}-$ средний за период поток энергии
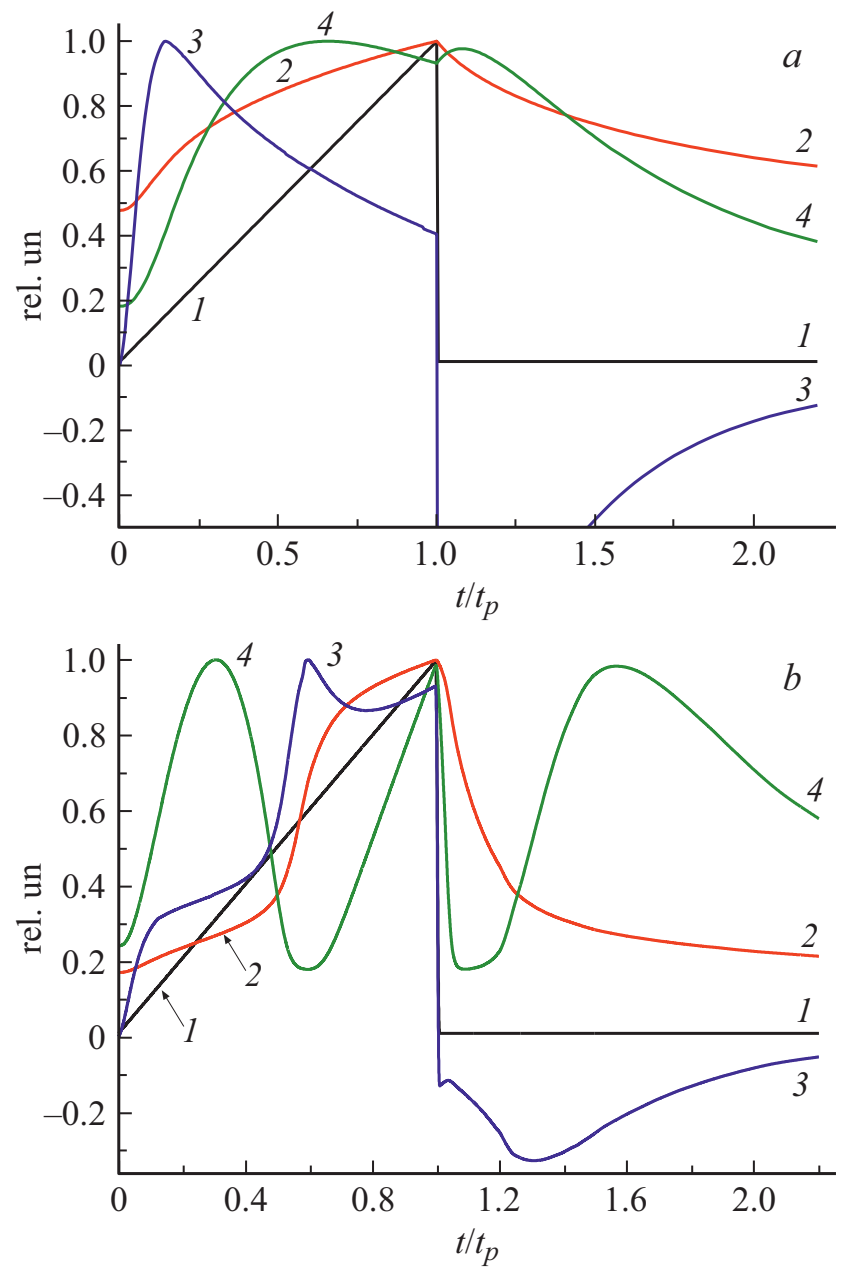

Рис. 1. Форма импульса тока $I(t)$ и зависимости от времени значений температуры $T_{0}(t)$, мощности нагрева $Q(t)$ и теплоемкости $c_{p}(t)$ на оси разряда. $1-I(t) / I_{\max }, 2-T_{0}(t) / T_{0} \max , 3-Q(t) / Q_{\max }$, $4-c_{p}(t) / c_{p \max } . a-p_{\text {sat }}=500$ Torr, $T_{0 \max }=6000 \mathrm{~K}$, $Q_{\max }=21940 \mathrm{~W} \cdot \mathrm{cm}^{-3}, \quad c_{p \max }=1391 \mathrm{~J} \cdot \mathrm{kg}^{-1} \cdot \mathrm{K}^{-1} ; \quad b-$ $p_{\text {sat }}=75$ Torr, $\quad T_{0 \max }=17610 \mathrm{~K}, \quad Q_{\max }=17980 \mathrm{~W} \cdot \mathrm{cm}^{-3}$, $c_{p \max }=1778 \mathrm{~J} \cdot \mathrm{kg}^{-1} \cdot \mathrm{K}^{-1}$.

излучения, поглощаемый стенками газоразрядной трубки [6].

Результаты моделирования ИПР в цезии в соответствии с (1)-(8) приведены для двух значений давления цезия: $p_{\text {sat }}=500$ Torr (рис. $\left.1, a-3, a\right)$ и 75 Torr (рис. $1, b-3, b)$, что соответствует массе цезия на единицу длины трубки $M=0.06$ и $0.009 \mathrm{mg} \cdot \mathrm{cm}^{-1}$. Для вычисления удельной теплоемкости плазмы $c_{p}$ использовано соотношение $c_{p}=(\partial H / \partial T)_{p=\text { const }}$, где $H=\left(2.5 p+n_{a} E_{a}+n_{i} E_{i}\right) / \rho-$ удельная энтальпия, $\rho=m_{a}\left(n_{a}+n_{i}\right)-$ плотность плазмы. Потери энергии на излучение $W_{v i s}(r, t)$ в видимой области спектра $\lambda=400-700 \mathrm{~nm}$ рассчитывались, как и $W_{\text {rad }}$, методом из работы [14].

Как видно из рис. $1, a$, в случае достаточно высокого начального давления температура $T_{0}$ и теплоемкость 
плазмы $c_{p}$ на оси разряда в течение импульса плавно возрастают. При этом реализуется центрированная структура плазменного канала (рис. 2,a) и бо́льшая часть видимого излучения выходит из приосевой области (рис. 3,a). Если давление перед импульсом невелико (рис. $1, b)$, то $T_{0}$ возрастает быстрее и наступает момент, когда вблизи оси разряда практически все атомы газа однократно ионизованы, а вторая ионизация и возбуждение ионов еще несущественны. Отметим, что такая ситуация реализуется благодаря большой разнице в значениях энергии первой и второй ионизации у атомов щелочных металлов. В это время на оси разряда теплоемкость $c_{p}$ плазмы резко уменьшается, а величина $Q=\sigma E^{2}-W_{\text {rad }}$, определяющая мощность нагрева плазмы, наоборот, возрастает (рис. 1, $b$, $\left.t / t_{p}=0.6\right)$. Это приводит к быстрому росту температуры в приосевой области и выталкиванию плазмы в более холодную периферийную область (рис. 2,b). Формируется оболочечная структура плазменного канала, когда бо́льшая часть плазмы сосредоточена на периферии разряда. Бо́льшая часть видимого излучения
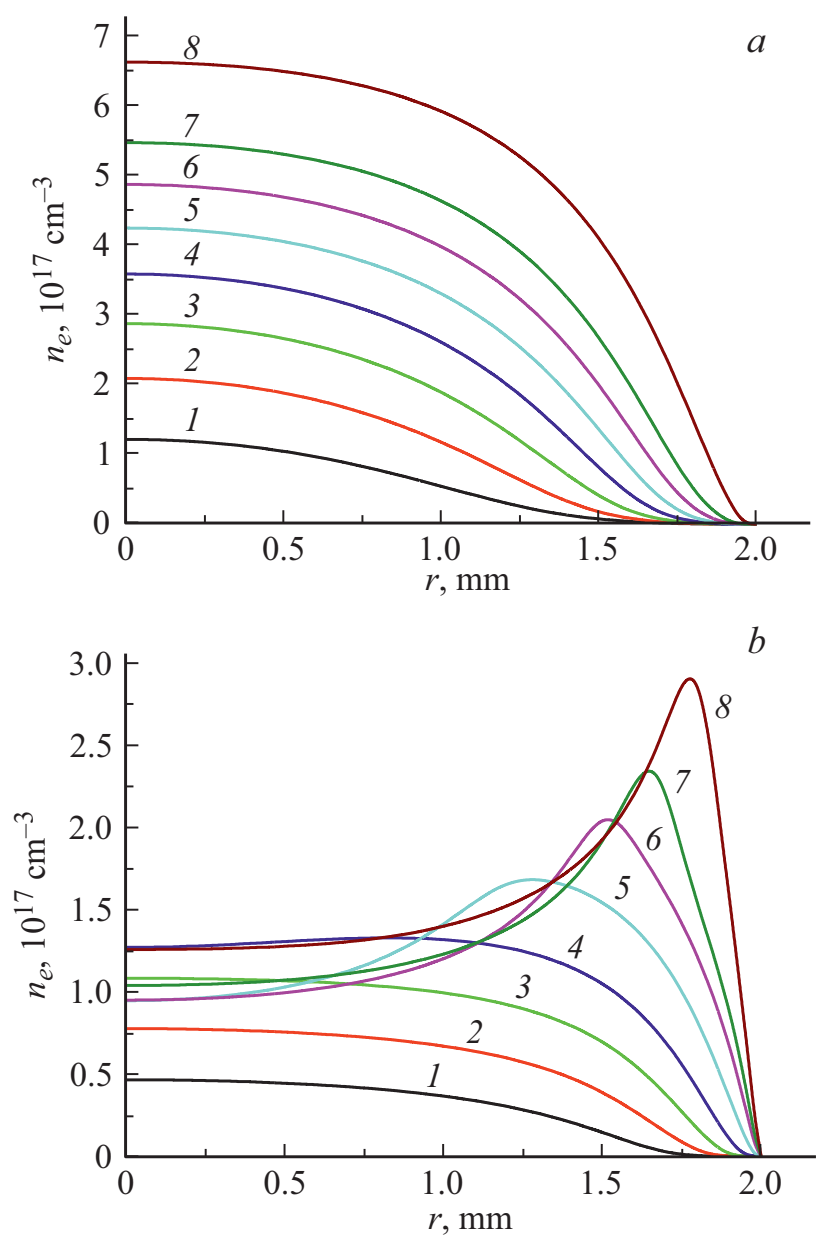

Рис. 2. Радиальные профили концентрации электронов $n_{e}(r, t)$ в различные моменты времени $t$ от начала импульса тока. $t, \mu \mathrm{s}$ : $1-10,2-15,3-20,4-25,5-30,6-35,7-40$, $8-50 . p_{\text {sat }}=500(a)$ и 75 Torr $(b)$.
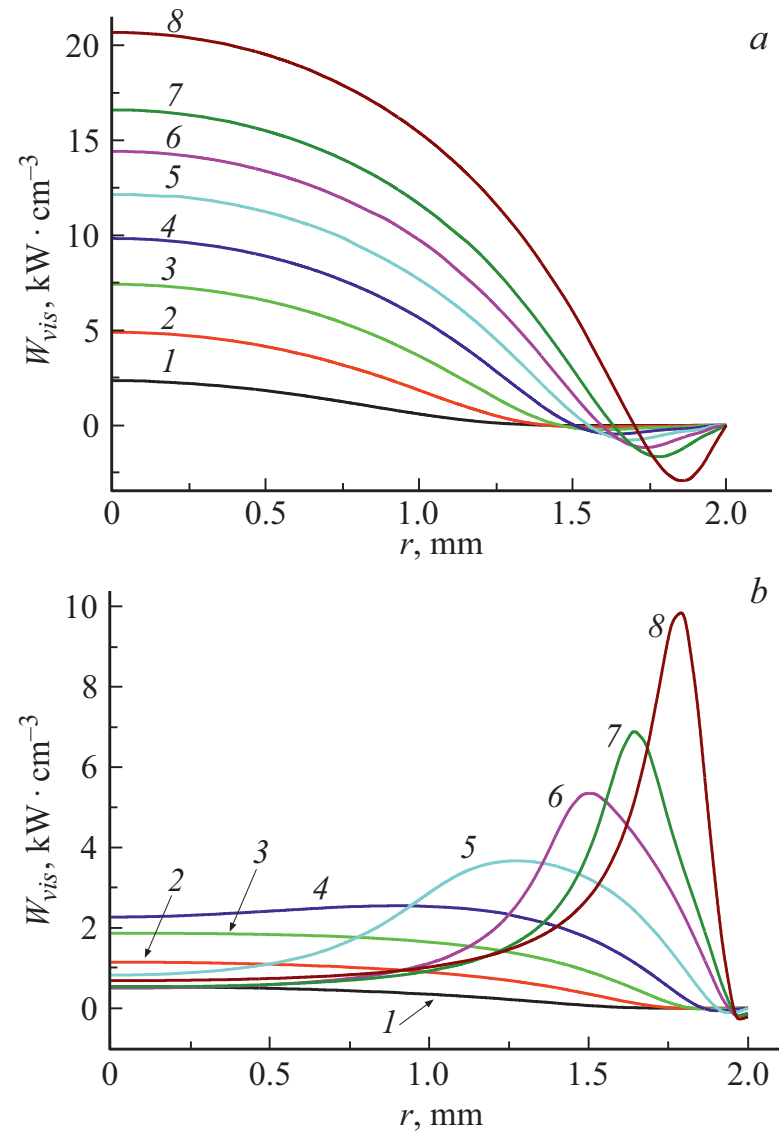

Рис. 3. Радиальное распределение радиационных потерь энергии $W_{v i s}(r, t)$ в видимой области в различные моменты времени $t$ от начала импульса тока. $t, \mu \mathrm{s}: 1-10,2-15,3-20$, $4-25,5-30,6-35,7-40,8-50 . p_{\text {sat }}=500(a)$ и 75 Torr $(b)$.

выходит теперь из плотной внешней оболочки канала (рис. $3, b)$. Переход от оболочечной к центрированной структуре канала сопровождается резким уменьшением радиуса светящегося канала разряда, что наблюдалось в работах [7,9]. Отметим, что после прохождения импульса тока в процессе остывания плазмы значение $c_{p}$ в приосевой области снова проходит через глубокий минимум и в разряде происходит обратная трансформация пространственного распределения плазмы.

Таким образом, в импульсно-периодическом разряде в цезии в зависимости от начального давления и амплитуды импульса тока может реализоваться центрированная либо оболочечная структура плазменного канала. Трансформация одной структуры в другую происходит в момент, когда теплоемкость плазмы достигает глубокого минимума, соответствующего полностью однократно ионизованной $e-i$-плазме.

\section{Конфликт интересов}

Авторы заявляют, что у них нет конфликта интересов. 


\section{Список литературы}

[1] Ю.С. Протасов, Энциклопедия низкотемпературной плазмы, под ред. акад. В.Е. Фортова (Наука, М., 2000), т. IV (вводный), с. 232-262.

[2] А.А. Рухадзе, А.Ф. Александров, Физика сильноточных электроразрядных источников света (ЛИБРОКОМ, М., 2012), c. 81-89.

[3] F.G. Baksht, V.F. Lapshin, J. Phys. D: Appl. Phys., 41 (20) 205201 (2008). DOI: 10.1088/0022-3727/41/20/205201

[4] M. Rakić, G. Pichler, JQSRT, 151, 169 (2015). DOI: $10.1016 /$ j.jqsrt.2014.09.022

[5] С.В. Гавриш, В.Б. Каплан, А.М. Марциновский, И.И. Столяров, Письма в ЖТФ, 41 (14), 64 (2015). [S.V. Gavrish, V.B. Kaplan, A.M. Martsinovskii, I.I. Stolyarov, Tech. Phys. Lett., 41 (7), 694 (2015).

DOI: $10.1134 / \mathrm{S} 1063785015070196]$

[6] Ф.Г. Бакшт, В.Ф. Лапшин, Прикладная физика, № 6, 5 (2019).

[7] С.В. Гавриш, В.Б. Каплан, А.М. Марциновский, И.И. Столяров, Прикладная физика, № 5, 78 (2019).

[8] Ф.Г. Бакшт, В.Ф. Лапшин, Прикладная физика, № 6, 10 (2020).

[9] А.А. Богданов, С.В. Гавриш, В.В. Коваль, А.М. Марциновский, И.И. Столяров, Прикладная физика, № 6, 16 (2020).

[10] А.А. Радциг, Б.М. Смирнов, Параметры атомов и атомных ионов: Справочник (Энергоатомиздат, М., 1986), c. 109.

[11] В.Н. Очкин, Спектроскопия низкотемпературной плазмы (Физматлит, М., 2010), с. 20-21, 60-68.

[12] В.Е. Фортов, А.Г. Храпак, И.Т. Якубов, Физика неидеальной плазмы (Физматлит, М., 2004), с. 137-138.

[13] В.М. Жданов, Процессы переноса в многокомпонентной плазме (Физматлит, М., 2009), с. 177-178.

[14] V.F. Lapshin, J. Phys.: Conf. Ser., 669 (1), 012035 (2016). DOI: $10.1088 / 1742-6596 / 669 / 1 / 012035$

[15] G.C. Wei, J. Phys. D: Appl. Phys., 38 (17), 3057 (2005). DOI: $10.1088 / 0022-3727 / 38 / 17 / \mathrm{S} 07$ 